\title{
Angiotensin-Converting Enzyme Activity is Involved in the Mechanism of Increased Endogenous Nitric Oxide Synthase Inhibitor in Patients With Type 2 Diabetes Mellitus
}

\author{
Akira Ito, MD; Kensuke Egashira, MD*; Takahiro Narishige, MD; \\ Kouhei Muramatsu, MD; Akira Takeshita, MD*
}

\begin{abstract}
The renin-angiotensin system plays an important role in the elevation of asymmetric dimethylarginine (ADMA), an endogenous inhibitor of nitric oxide synthase, in hypertensive patients, so the present study was designed to examine whether angiotensin-converting enzyme (ACE) activity is also involved in the mechanism of ADMA elevation in type 2 diabetes mellitus (NIDDM). A crossover study was performed to determine if ACE inhibition with perindopril $(4 \mathrm{mg} / \mathrm{day})$ for 4 weeks decreases serum ADMA concentration and plasma von Willebrand factor (vWF) level (a marker of endothelial injury) in 11 patients with NIDDM. None of the patients was treated with insulin or oral hypoglycemic drugs, and none had major diabetic complications. Before the protocol began, serum ADMA and plasma vWF were significantly higher in the 11 NIDDM patients, when compared with 8 control subjects without diabetes. Perindopril did not affect blood pressure or glucose metabolism, but did significantly decrease serum ADMA and plasma vWF. These results suggest that endothelial injury associated with ADMA elevation may be present even in patients with non-complicated NIDDM, and that increased activity of ACE may be involved in such endothelial dysfunction. (Circ J 2002; 66: 811-815)
\end{abstract}

Key Words: Angiotensin-converting enzyme inhibitor; Asymmetric dimethylarginine; Endothelial injury; Noninsulin dependent diabetes mellitus; von Willebrand factor

C ardiovascular disease is the leading cause of mortality in patients with diabetes mellitus (DM). Although established macroangiopathy is characterized by morphological changes of the arterial wall typical of atherosclerosis, it has been suggested that the earliest phase of atherogenesis is caused by dysfunction of the vascular endothelium!

Endothelium-derived nitric oxide (NO) is a potent vasodilator that plays a critical role in regulating vascular resistance and flow, as well as inhibiting key processes in atherogenesis, such as monocyte adhesion, platelet aggregation and vascular smooth muscle proliferation? In disorders associated with atherosclerosis (eg hypertension, hypercholesterolemia, DM), there is less endotheliumdependent NO-mediated vasodilation? Impairment of the NO synthase system adversely affects vascular reactions and blood flow and, in addition, because NO inhibits key processes in atherogenesis, an NO deficiency state may contribute to the initiation and the progression of atherosclerosis 3,4

Although the mechanisms of endothelial vasodilator dysfunction are likely multifactorial, one contributing abnormality appears to be increased levels of asymmetric dimethylarginine (ADMA),6 ADMA is an endogenous

(Received March 18, 2002; revised manuscript received May 20, 2002; accepted Jnue 7, 2002)

Department of Cardiovascular Medicine, Yamaguchi Red Cross Hospital, Yamaguchi and *Department of Cardiovascular Medicine, Graduate School of Medicine, Kyushu University, Fukuoka, Japan

Mailing address: Akira Ito, $\mathrm{MD}, \mathrm{PhD}$, Department of Cardiovascular Medicine, Yamaguchi Red Cross Hospital, 53-1 Yawatanobaba, Yamaguchi 753-8519, Japan. E-mail: iakira@ mbc.sphere.ne.jp competitive inhibitor of NO synthase (NOS) 7 and serum or plasma levels of ADMA are elevated in individuals with hypertension, hypercholesterolemia, $\mathrm{DM}^{6}$, peripheral arterial occlusive disease ${ }^{9}$ or congestive heart failure ${ }^{10}$ Elevation of ADMA is associated with impaired endothelium-dependent NO-mediated vasodilation in the brachial artery ${ }^{5}$ and is also significantly correlated with the intimamedia thickness of the carotid artery, a noninvasive measure of atherosclerosis. ADMA is thought to derive from proteins that have been posttranslationally methylated and subsequently hydrolyzed to release ADMA $!^{11}$ A number of cells elaborate ADMA, including vascular endothelial cells,$^{12}$ and it may be excreted in the urine or metabolized by the enzyme dimethylarginine dimethylaminohydrolase $(\mathrm{DDAH})^{13}$ to L-citrulline and dimethylamine. We recently reported that dysregulation of DDAH induced by lipids ${ }^{14}$ or hyperglycemia ${ }^{15}$ may play an important role in the elevation of ADMA in hypercholesterolemia or DM, respectively. However, it is not well understood how the serum concentration of ADMA becomes elevated in humans in vivo.

It has been reported that angiotensin-converting enzyme (ACE) inhibitors improve endothelial function in patients with hypertension ${ }^{16}$ or $\mathrm{DM}{ }^{17}$ suggesting that the activation of the renin-angiotensin system (RAS) may contribute to the endothelial dysfunction in those individuals. We have recently demonstrated that RAS plays an important role in the elevation of ADMA as well as endothelial injury in hypertensive patients 18 Therefore, we investigated whether ACE activity is involved in the mechanisms of endothelial injury associated with ADMA elevation in patients with type 2 DM (NIDDM). 
Table 1 Clinical Characteristics of Patients With Noninsulin-Dependent Diabetes Mellitus and Control Subjects

\begin{tabular}{lccc}
\hline \hline & NIDDM $(n=11)$ & Control $(n=8)$ & p value \\
\hline Age (years) & $74 \pm 2$ & $71 \pm 2$ & $N S$ \\
M/F & $5 / 6$ & $4 / 4$ & $N S$ \\
Mean blood pressure $(\mathrm{mmHg})$ & $97 \pm 3$ & $93 \pm 4$ & $N S$ \\
Total cholesterol $(\mathrm{mg} / \mathrm{dl})$ & $198 \pm 7$ & $209 \pm 14$ & $N S$ \\
Triglyceride $(\mathrm{mg} / \mathrm{dl})$ & $114 \pm 15$ & $148 \pm 23$ & $N S$ \\
Fasting blood sugar $(\mathrm{mg} / \mathrm{dl})$ & $132 \pm 7$ & $89 \pm 3$ & $<0.001$ \\
Glycated hemoglobin $(\%)$ & $6.8 \pm 0.3$ & - & - \\
Urea nitrogen $(\mathrm{mg} / \mathrm{dl})$ & $16 \pm 1$ & $15 \pm 2$ & $N S$ \\
Creatinine $(\mathrm{mg} / \mathrm{dl})$ & $0.74 \pm 0.08$ & $0.79 \pm 0.12$ & $N S$ \\
ADMA $\mu$ mol/L) & $0.53 \pm 0.03$ & $0.42 \pm 0.01$ & $<0.01$ \\
vWF $(\%)$ & $219 \pm 22$ & $146 \pm 15$ & $<0.05$ \\
Lipoperoxide $\mu \mathrm{mol} / \mathrm{L})$ & $3.0 \pm 0.2$ & $3.0 \pm 0.4$ & $N S$ \\
\hline
\end{tabular}

ADMA, asymmetric dimethylarginine; $v W F$, von Willebrand factor. NS, not significant.

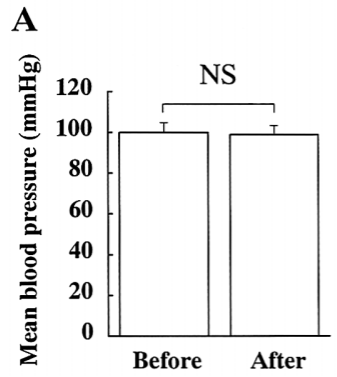

B

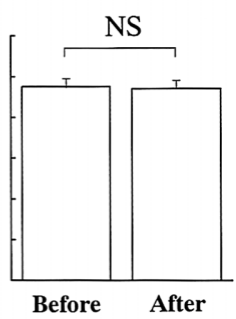

Fig 1. Changes of mean blood pressure by 4 week treatment with (A) or without (B) add-on perindopril. NS, not significant.
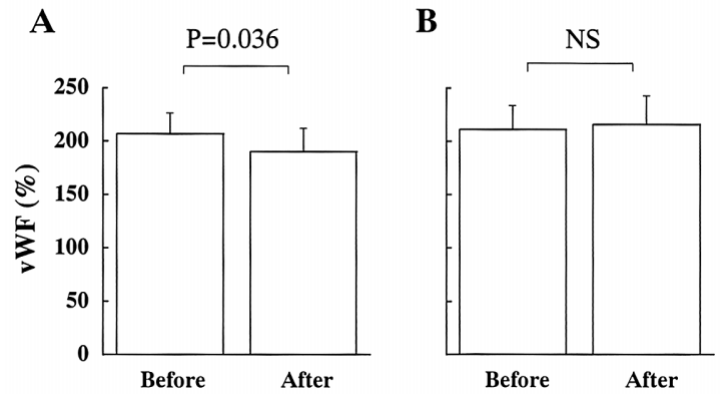

Fig 3. Changes of plasma vWF concentration by 4 week treatment with (A) or without (B) add-on perindopril. vWF, von Willebrand factor. NS, not significant.

\section{Methods}

\section{Patients}

The study was performed in 11 patients with NIDDM, 8 of whom had been treated with antihypertensive agents other than ACE inhibitors or angiotensin II type 1 receptor blockers, and 8 patients without DM (4 treated for chronic atrial fibrilation (AF) with warfarin and digitalis; paroxysmal AF treated with cibenzoline in 1; essential hypertension treated with amlodipine or atenolol in 3). All the patients gave informed consent to be enrolled in the study, which has been approved by the hospital's Ethics Committee. None of the NIDDM patients was treated with insulin or oral hypoglycemic drugs, and none had major diabetic complications, such as retinopathy, nephropathy, neuropathy, history of stroke, suggestive coronary artery disease or peripheral arterial occlusive disease. The 8 pa-
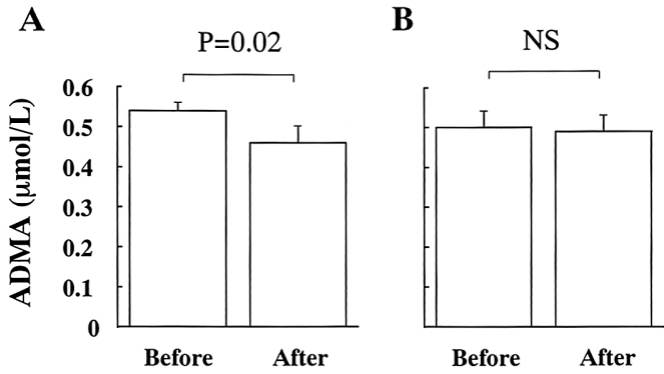

Fig 2. Changes of serum ADMA concentration by 4 week treatment with (A) or without (B) add-on perindopril. ADMA, asymmetric dimethylarginine; NS, not significant.
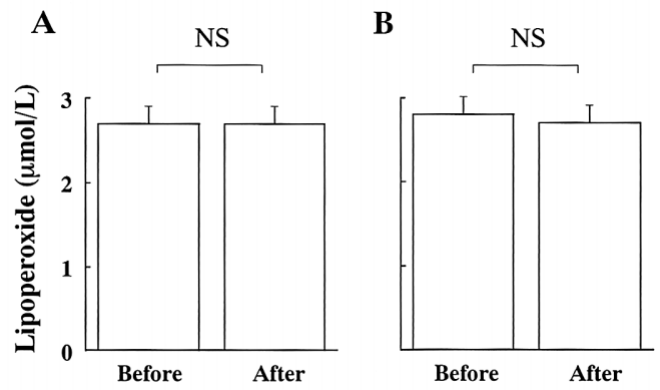

Fig 4. Changes of serum lipoperoxide concentration by 4 week treatment with (A) or without (B) add-on perindopril. NS, not significant.

tients with NIDDM who were treated with antihypertensive agents ( 6 with a calcium channel blocker, 1 with a $\beta$-blocker and 1 with a combination of the both drugs) had well controlled blood pressure (BP): systolic BP $<140 \mathrm{mmHg}$ and diastolic $\mathrm{BP}<90 \mathrm{mmHg}$.

\section{Measurements}

The following measurements were performed in all the patients.

(1) BP was measured in the seated positions after several deep breaths.

(2) Serum ADMA concentration was measured by highperformance liquid chromatography. The variability of the method was less than $7 \%$, and the detection limit of the assay was $0.15 \mathrm{mmol} / \mathrm{L}$.

(3) Plasma concentration of von Willebrand factor 
(vWF, a marker of endothelial injury) was measured by an enzyme immunoassay technique. The within-run coefficient of variation was $2-3 \%$. It has been reported that an increased level of vWF is associated with impaired endothelium-dependent vasodilation. ${ }^{19}$

(4) Serum lipoperoxide was also determined as a marker of oxidative stress by spectrophotometric measurement of thiobarbituric acid reactivity.

\section{Study Protocol}

Five NIDDM patients were treated with add-on perindopril ( $4 \mathrm{mg} /$ day) for the first 4 weeks, and then the preceding measurements were repeated. Perindopril was stopped for 4 weeks, and then the measurements were repeated again and after the second 4 week interval. The other 6 NIDDM patients were treated without add-on perindopril for the first 4 weeks, and after a 4 week interval, add-on perindopril was started for 4 weeks. The measurements were performed before and after the first and the second 4-week treatment.

\section{Statistical Analysis}

Values are expressed as mean \pm SE. Differences between NIDDM patients and patients without DM were tested by unpaired Students' $t$ test. Between before and after additional perindopril or no additional treatment, differences were evaluated by paired Students' t test; $\mathrm{p}<0.05$ was considered statistically significant.

\section{Results}

The clinical characteristics of the patients are shown in Table 1. There were no significant differences between NIDDM patients at baseline and patients without DM (controls) in age, sex, BP, serum cholesterol, triglyceride, urea nitrogen or creatinine levels. Although the fasting blood sugar (FBS) level was significantly elevated in NIDDM patients, the blood sugar control in these patients was good $(\mathrm{FBS}=132 \pm 7 \mathrm{mg} / \mathrm{dl}$ and glycated hemoglobin $=$ $6.8 \pm 0.3 \%$ ). Serum ADMA concentration and plasma vWF level were significantly elevated in NIDDM patients, when compared with the control. There was no significant difference between the 2 groups in serum lipoperoxide level.

In NIDDM patients, add-on perindopril for 4 weeks did not change BP (Fig 1A) or glucose metabolism (FBS = $132 \pm 7-134 \pm 9 \mathrm{mg} / \mathrm{dl}, \mathrm{p}=0.60)$. However, add-on perindopril significantly decreased serum ADMA concentration (Fig 2A) and plasma vWF level (Fig 3A). There were no significant changes during 4 week observation without addon perindopril in BP (Fig 1B), serum ADMA (Fig 2B) or plasma vWF (Fig 3B). There were no significant changes in serum lipoperoxide level with or without add-on perindopril (Fig 4A,B).

Urinary excretion of ADMA did not significantly change by add-on perindopril (urinary ADMA/urinary creatinine; $45 \pm 5-44 \pm 5 \mu \mathrm{mol} / \mathrm{mg}$ creatinine).

\section{Discussion}

The salient findings of this study are: (1) serum ADMA concentration and plasma vWF level (a marker of endothelial injury) were significantly elevated in patients with noncomplicated NIIDDM, and (2) serum ADMA and plasma vWF were significantly decreased by an ACE inhibitor (perindopril).

This study provides insight into a novel mechanism by which activation of ACE may disturb both the NOS pathway and endothelial function in NIDDM.

Increased levels of ADMA, the endogenous NOS inhibitor, are observed in individuals with $\mathrm{DM}^{6}$ and may account in part for the endothelial vasodilator dysfunction observed in this condition. Increased ADMA levels are associated with reduced NO elaboration in both hypercholesterolemic ${ }^{5}$ and atherosclerotic patients, as judged by reduced nitrate excretion and impaired endothelium-dependent, NO-mediated forearm vasodilation.

\section{Elevation of ADMA and $v W F$ in NIDDM}

In the present study, the serum ADMA and plasma vWF concentrations were significantly elevated in patients with noncomplicated NIDDM, in whom the blood sugar control was good without insulin or oral hypoglycemic drugs, when compared with control subjects without DM, suggesting that endothelial injury associated with ADMA elevation might be present even in these patients. There was no significant difference between NIDDM patients and controls in BP, with or without antihypertensive agents other than ACE inhibitors or angiotensin II receptor blockers. In 8 control subjects without DM, 4 were treated for chronic AF with warfarin. It has been reported that plasma vWF concentration is elevated in patients with chronic AF, and that anticoagulation with warfarin has no effect ${ }^{20}$ In the present study, however, the plasma vWF concentration in NIDDM patients was further elevated when compared with control subjects without DM including patients with chronic AF. Although antihypertensive treatment is known to improve endothelial function ${ }^{21}$ these effects of antihypertensive treatment on the difference of plasma vWF concentration between NIDDM patients and control subjects without DM were negligible in the present study, because hypertensive patients both in NIDDM $(n=8)$ and control group $(n=3)$ were treated with the same kind of agents (calcium channel blockers and/or $\beta$-blockers), and because the BP control was comparable between the 2 groups.

\section{Effects of ACE Inhibition on ADMA and $v W F$}

Four-week treatment with add-on perindopril did not significantly change BP in NIDDM patients. The dose of perindopril in the present study ( $4 \mathrm{mg} /$ day) is routinely used for antihypertensive treatment and known to decrease BP. We somewhat surprisingly found no change in BP with this usual dose of perindopril. The mechanism of no reduction in BP with perindopril in the present study is not understood, although several studies have reported that chronic treatment with ACE inhibitors did not change BP in wellcontrolled hypertensive or normotensive patients with DM ${ }^{22-24}$ Even in hypertensive patients, almost $30 \%$ of the patients in one study were non-responders (no significant fall in BP) to ACE inhibitors and that the fall in BP by ACE inhibitors was related to pre-treatment plasma renin activity 25 However, we did not measure plasma renin activity, serum ACE activity or plasma angiotensin II level in this study. Further studies are needed to clarify the mechanisms of the nonreduction in BP with ACE inhibition in wellcontrolled hypertensive or normotensive patients. The present result that perindopril did not change BP suggests that the perindopril-induced decrease of serum ADMA and plasma vWF was not caused by the antihypertensive action of this drug. Our previous study also demonstrated that perindopril, but not bisoprolol, significantly decreased 
serum ADMA and plasma vWF concentrations in patients with essential hypertension, although these 2 drugs decreased BP to the same extent ${ }^{18}$

\section{Mechanism of ADMA Elevation in NIDDM}

We recently demonstrated that dysregulation of DDAH, a degradation enzyme of ADMA, by lipids ${ }^{14}$ or hyperglycemia $^{15}$ plays an important role in the elevation of ADMA in hypercholesterolemia or DM, respectively. DDAH has sulfhydryl groups in its structure ${ }^{13}$ and oxidative stress has been suggested as one of the mechanisms of its decreased activity. ${ }^{6}$ ACE inhibitors have been shown to reduce free radical concentrations in patients with coronary artery disease ${ }^{27}$ In the present study, we measured serum lipoperoxide as a marker of oxidative stress, and perindopril did not change its serum concentration. Taken together with our previous finding that perindopril did not change serum malondialdehyde-modified low density lipoprotein (another marker of oxidative stress) ${ }^{18}$ it is possible that other mechanisms than the reduction of oxidative stress are involved in the perindopril-induced decrease of serum ADMA, although we measured only 2 crude markers of oxidative stress. Further studies are needed to clarify the role of DDAH in the effects of ACE inhibitors on serum ADMA levels in patients with NIDDM.

\section{Pathological Meaning of Serum ADMA Elevation in NIDDM}

There was no correlation between ADMA and vWF, or between the decrease of ADMA and $\mathrm{vWF}$, and the decrease of ADMA by perindopril was only $10-20 \%$. These results suggest that some factors other than those measured in this study, such as serum homocystein levels etc, might be related to endothelial function, and that the elevation of ADMA is just one of the key factors contributing to endothelial dysfunction. In the present study, circulating ADMA concentrations in NIDDM patients were less than $1 \mu \mathrm{mol} / \mathrm{L}$, although they were significantly higher than in control subjects without DM. It is known that concentrations of L-arginine (substrate for NOS) are in the order of $80 \mathrm{\mu} \mathrm{mol} / \mathrm{L}$ even in diseased states 28 At these concentrations of L-arginine, any competitive inhibition of NOS by ADMA would be overcome. However, it is also known that L-arginine supplementation can increase NO production by vascular endothelial cells under certain conditions? This phenomenon 'arginine paradox' occurs in spite of the apparently saturating concentration of L-arginine inside the cells. It is suggested that in endothelial cells, endothelial NOS (eNOS) is predominantly localized to a perinuclear region with a smaller but significant pool localized to caveolae on the plasma membrane ${ }^{29}$ ADMA enters cells through the cationic amino acid transporters known as a $\mathrm{y}^{+}$ transporter which also transports L-arginine ${ }^{28}$ The recent finding that a $\mathrm{y}^{+}$transporter co-locates with caveolin-bound eNOS suggests that activity of this transporter may be important to determine local concentrations of L-arginine and ADMA 30 One explanation of the 'arginine paradox' relates to the possibility of 'compartmentalization' of amino acid concentrations. The suggestion that intracellular Larginine is sequestered in storage pools which eNOS does not have access raises the possibility that L-arginine/ ADMA ratio in the immediate vicinity of the enzyme may be very different from the total cellular L-arginine/ADMA ratio, 28 and ADMA may produce biological effects at low concentrations as in our patients with NIDDM.

\section{Study Limitations}

There are some drawbacks in this study that need to be mentioned. First, the patient number was small. Further large-scaled studies are required to evaluate the effects of ACE inhibitors on circulating ADMA concentration and endothelial function in NIDDM patients. Second, we measured plasma vWF concentration as a marker of endothelial injury, which is thought to be increased in disorders with endothelial dysfunction, ${ }^{31}$ but did not evaluate endothelial function itself, for example, by measurement of flow-dependent vasodilation of brachial artery using an ultrasound technique? ${ }^{2}$

ADMA is widely distributed in tissues ${ }^{13}$ and may be the mechanism for controlling NO synthesis in physiological and/or pathological states. We have demonstrated that the ACE activity may be involved in the elevation of serum ADMA in NIDDM and our results suggest that the vasculoprotective actions of ACE inhibitors can be explained at least in part by the amelioration of endothelial injury (dysfunction) through the decreased serum ADMA concentration.

\section{References}

1. Semenkorich CF, Heinecke JW. The Mystery of diabetes and atherosclerosis: Time for a new plot. Diabetes 1997; 46: 327-334.

2. Furchgot RF, Zawadzki JV. The obligatory role of endothelial cells in the relaxation of arterial smooth muscle by acetylcholine. Nature 1980; 288: $373-376$

3. Cooke JP, Dzau VJ. Derangements of the nitric oxide synthase pathway, L-arginine, and cardiovascular diseases. Circulation 1997; 96: $379-382$.

4. Takemoto M, Egashira K, Usui M, Numaguchi K, Tomita H, Tsutsui $\mathrm{H}$, et al. Important role of tissue angiotensin-converting enzyme activity in the pathogenesis of coronary vascular and myocardial structural changes induced by long-term blockade of nitric oxide synthesis in rats. J Clin Invest 1997; 99: 278-287.

5. Boeger RH, Bode-Boeger SM, Szuba A, Tsao PS, Chan JR, Tangphao O, et al. Asymmetric dimethylarginine (ADMA): A novel risk factor for endothelial dysfunction: Its role in hypercholesterolemia. Circulation 1998; 98: $1842-1847$.

6. Miyazaki H, Matsuoka H, Cooke JP, Usui M, Ueda S, Okuda S, et al. Endogenous nitric oxide synthase inhibitor: A novel marker of atherosclerosis. Circulation 1996; 99: 1141-1146.

7. Vallance P, Leone AM, Calver A, Collier J, Moncada S. Endogenous dimethylarginine as an inhibitor of nitric oxide synthase. J Cardiovasc Pharmacol 1992; 20(Suppl 12): 560-562.

8. Surdacki A, Nowicki M, Sandmann J, Tsikas D, Boeger RH, BodeBoeger SM, et al. Reduced urinary excretion of nitric oxide metabolites and increased plasma levels of asymmetric dimethylarginine in men with essential hypertension. J Cardiovasc Pharmacol 1999; 33: $652-658$.

9. Boeger RH, Bode-Boeger SM, Thiele W, Junker W, Alexander K, Froelich JC. Biochemical evidence for impaired nitric oxide synthesis in patients with peripheral arterial occlusive disease. Circulation 1997; 95: 2068-2074.

10. Usui M, Matsuoka H, Miyazaki H, Ueda S, Okuda S, Imaizumi T. Increased endogenous nitric oxide synthase inhibitor in patients with congestive heart failure. Life Sci 1998; 62: 2425-2430.

11. Kakimoto $\mathrm{Y}$, Akazawa S. Isolation and identification of $\mathrm{N}^{\mathrm{G}}, \mathrm{NG}^{\mathrm{G}}$ and $\mathrm{N}^{\mathrm{G}}, \mathrm{N}^{\prime} \mathrm{G}$-dimethylarginine, Ne-mono-, di-, and trimethyllysine, and glucosylgalatosyl- and galactosyl-d-hydroxylysine from human urine. J Biol Chem 1970; 245: 5751-5758.

12. Fickling S, Leone AM, Nussey SS, Vallance P, Whitley GStJ. Synthesis of $\mathrm{N}^{\mathrm{G}}$, $\mathrm{N}^{\mathrm{G}}$ dimethylarginine by human endothelial cells. Endothelium 1993; 1: 137-140.

13. Kimoto M, Whitley GS, Tsuji H, Ogawa T. Detection of $\mathrm{N}^{\mathrm{G}}, \mathrm{NG}_{-}$ dimethylarginine dimethylaminohydrolase in human tissues using a monoclonal antibody. J Biochem 1995; 117: 237-238.

14. Ito A, Adimoolam S, Tsao PS, Kimoto M, Ogawa T, Cooke JP. Novel mechanism for endothelial dysfunction: Dysregulation of dimethyarginine dimethylaminohydrolase. Circulation 1999; 99: 3092 3095 .

15. Ito A, Asagami T, Tsao PS, Adimoolam S, Kimoto M, Tsuji H, et al. A novel mechanism of endothelial dysfunction in diabetes mellitus 
(abstract). Circulation 1999; 100(Suppl I): I-473.

16. Rizzoni D, Muiesan ML, Porteri E, Castellano M, Zulli R, Bettoni G, et al. Effects of long-term therapy with lisinopril on resistance arteries of hypertensive patients with left ventricular hypertrophy. $J$ Hypertens 1997; 15: 197-204.

17. O'Driscoll G, Green D, Marionara A, Stanton K, Colreavy F, Taylor $\mathrm{R}$. Improvement in endothelial function by angiotensin-converting enzyme inhibitor in non-insulin-dependent diabetes mellitus. $J \mathrm{Am}$ Coll Cardiol 1999; 33: 1506-1511.

18. Ito A, Egashira K, Narishige T, Muramatsu K, Takeshita A. Reninangiotensin system is involved in the mechanism of increased serum asymmetric dimethylarginine in essential hypertension. Jpn Circ J 2001; 65: 775-778.

19. Blann AD. von Willebrand factor and the endothelium in vascular disease. Br J Biomed Sci 1993; 50: 125-134.

20. Li-Saw-Hee FL, Blann AD, Lip GY. Effects of low-dose warfarin, aspirin-warfarin combination therapy, and dose-adjusted warfarin on thrombogenesis in chronic atrial fibrillation. Stroke 2000; 31: 828 833.

21. Taddei S, Virdis A, Ghiadoni L, Sudano I, Salvetti A. Effects of antihypertensive drugs on endothelial dysfunction: Clinical implications. Drugs 2002; 62: 265-284.

22. Sano T, Kawamura T, Matsumae H, Sasaki H, Nakayama M, Hara T, et al. Effects of long-term enalapril treatment on persistent microalbuminuria in well-controlled hypertensive and normotensive NIDDM patients. Diabetes Care 1994; 17: 420-424.

23. Parving HH, Hommel E, Jensen BR, Hansen HP. Long-term beneficial effects of ACE inhibition on diabetic nephropathy in normotensive type 1 diabetic patients. Kidney Int 2001; 60: 228-234.

24. Bojestig M, Lindstrom T, Karlberg BE, Nystrom FH. Reduction of ACE activity is insufficient to decrease microalbuminuria in normo- tensive patients with type 1 diabetes. Diabetes Care 2001; 24: 919924.

25. Sever PS, Chang CL. Discordant responses to two classes of drugs acting on the rennin-angiotensin system. J Renin Angiotensin Aldosterone Syst 2001; 2: 25-30.

26. Stuehlinger MC, Tsao PS, Kimoto M, Cooke JP. Homocysteine induced accumulation of asymmetric dimethylarginine: Role of DDAH and effect of antioxidants (abstract). Circulation 2000; 102(Suppl): II-177.

27. Berry C, Anderson N, Hamilton LA, Pathi V, Kirk AJB, McMurray $\mathrm{JJ}$, et al. Renin-angiotensin system inhibition reduces free radical concentrations in arteries of patients with coronary heart disease (abstract). Circulation 2000; 102(Suppl): II-123.

28. Leiper J, Vallance P. Biological significance of endogenous methylarginines that inhibit nitric oxide synthase. Cardiovasc Res 1999; 43: $542-548$.

29. Lui J, Gareia-Cardenn G, Sessa WC. Palmitoylarion of endothelial nitric oxide synthase is necessary for optimal stimulated release of nitric oxide: Implications for caveolae localization. Biochemistry 1996; 35: 13277-13281.

30. McDonald KK, Zharikov S, Block ER, Kilberg MS. A caveolar complex between the cationic amino acid transporter I and endothelial nitric oxide synthase may explain the 'arginine paradox'. J Biol Chem 1997; 272: 31213-31216.

31. Lip GYH, Blann A. von Willebrand factor: A marker of endothelial dysfunction in vascular disorders? Cardiovasc Res 1997; 34: $253-$ 265.

32. Celermajer DS, Sorensen KE, Gooch VM, Spiegelhalter DJ, Miller OI, Sullivan ID, et al. Non-invasive detection of endothelial dysfunction in children and adults at risk of atherosclerosis. Lancet 1992; 340: $1111-1115$. 